\title{
Recognition of false alarms in fall detection systems
}

\author{
Stefano Abbate*, Marco Avvenuti ${ }^{\dagger}$, Guglielmo Cola, Paolo Corsini ${ }^{\dagger}$, Janet Light ${ }^{\ddagger}$ and Alessio Vecchio ${ }^{\dagger}$ \\ *IMT Institute for Advanced Studies, Lucca, Italy, stefano.abbate@imtlucca.it \\ ${ }^{\dagger}$ Dip. di Ingegneria dell’Informazione, Università di Pisa, Pisa, Italy, firstname.lastname@iet.unipi.it \\ $\ddagger$ Dep. of Computer Science \& Applied Statistics, Univ. of New Brunswick, Saint John, Canada, jlight@unb.ca
}

\begin{abstract}
Falls are a major cause of hospitalization and injuryrelated deaths among the elderly population. The detrimental effects of falls, as well as the negative impact on health services costs, have led to a great interest on fall detection systems by the health-care industry. The most promising approaches are those based on a wearable device that monitors the movements of the patient, recognizes a fall and triggers an alarm. Unfortunately such techniques suffer from the problem of false alarms: some activities of daily living are erroneously reported as falls, thus reducing the confidence of the user. This paper presents a novel approach for improving the detection accuracy which is based on the idea of identifying specific movement patterns into the acceleration data. Using a single accelerometer, our system can recognize these patterns and use them to distinguish activities of daily living from real falls; thus the number of false alarms is reduced.
\end{abstract}

\section{BACKGROUND}

For the elderly population, falls are a frequent cause of severe injury. Thus, the presence of an automated system that is able to detect falls can improve the caregiving process and the quality of life among senior citizens. In the remaining part of this section, recent techniques for the detection of falls are introduced and discussed, and the motivation for further improvements is presented.

\section{A. Requirements}

In order to be useful, a fall detection system must satisfy the following requirements:

- Automation: the systems activated by pushing a button are not satisfactory, since after a fall the persons may be unable to ask for help.

- Reactivity: fast detection of falls is critical, as it has been established that earlier the fall is reported, lower is the rate of disability/mortality.

- Accuracy: the system must offer the greatest degree of accuracy in detecting actual falls as well as in filtering false alarms. Two criteria of quality can be defined [9]:

- Sensitivity: capacity to detect actual falls. Defined as the ratio between the number of falls properly detected (true positives) and the falls that actually happened (true positives plus false negatives).

- Specificity: capacity to filter false alarms. Defined as the ratio between fall-like actions properly discarded (true negatives) and the total number of discarded actions (true negatives plus false positives).

The highest degree of accuracy is reached when both sensitivity and specificity values are equal to 1 (or 100\%), meaning that the system always detects actual falls and never produces false alarms.

- Usability: the monitoring method should be almost invisible to patients and should not pose privacy concerns.

\section{B. Methods for the detection of falls}

Fall detection methods can be grouped as follows [10]:

1) Camera based: Cameras are installed in the rooms to be monitored. Acquired data is then processed using proper algorithms, which can be divided into three main categories: i) inactivity detection: based on the principle that a person after falling remains inactive for some time; $i i$ ) shape change analysis: based on the change of posture during a fall; iii) 3D head motion analysis: head position and speed are monitored to recognize alarm conditions.

The advantages of this approach are: the ability to detect more events simultaneously, the low level of intrusiveness and the fact that recorded data can be easily used for remote verification. On the other hand, the limitations are represented by costs and time required for installation as well as privacy concerns for people being monitored. Moreover, detection is strictly limited to the places equipped with cameras.

2) Ambient device: Multiple sensors are installed in the places to be monitored, in order to acquire some kind of information when people are close to them. Common examples are pressure sensors on the floor, bed exit detectors and IR sensor arrays on the walls. Collected data are then analyzed to determine if a fall has happened.

This approach uses cheap and non-intrusive devices. However, installation may require some time and detection is strictly limited to the area equipped with sensors.

3) Wearable device: The patient wears a smart device which is generally equipped with sensors such as accelerometers and gyroscopes, to acquire kinematic and posture information. A first evaluation of sensed data can be done on the device itself using embedded intelligence, while for further elaboration collected data are generally sent to a base station by means of wireless communication. This solution is somehow more intrusive for patients, as it requires to wear at least a device. Nevertheless, it offers important benefits such as fast set-up operations, low cost, and it poses small privacy concerns, especially when compared with camerabased solutions.

From now on we will focus on the wearable approach. More precisely, we will consider only systems that use the analysis of motion data for the detection of falls. 


\begin{tabular}{|l|l|}
\hline Sensor(s) position & Identified postures \\
\hline chest & (standing or sitting), (bending or lying) \\
\hline waist & (bending or standing or sitting), (lying) \\
\hline chest+thigh & bending, lying, standing, sitting \\
\hline
\end{tabular}

TABLE I

POSTURES WHICH CAN BE IDENTIFIED DEPENDING ON SENSOR(S) POSITION.

\section{State of the art of fall detection methods using wearable devices}

In recent scientific literature, it is possible to find many attempts to solve the fall detection problem using one or both of the following types of sensors:

- Accelerometer: it measures the linear acceleration values on the different axes. The obtained measure is $0 g$ when an axis is orthogonal to the gravity or in case of free fall, while it is $1 g$ when the device is not moving and the considered axis has same direction as the gravity.

- Gyroscope: it measures the angular velocity on each axis. These sensors are usually quite power consuming with respect to accelerometers, microcontrollers, and transceivers.

Fall detection methods can be characterized considering how kinetic data is used to distinguish "activities of dailyliving" (ADLs) from harmful falls.

1) Fixed threshold approach: In this case a fixed kinematic threshold is used to determine if a fall has happened. In [3] the authors describe a system based on the magnitude of acceleration values, while [2] presents a technique based on measures of the angular velocity obtained from gyroscopes. The critical issue in this approach is the definition of a proper threshold: if the value is too high the system may miss some real falls (sensitivity $<100 \%$ ) but never generates false alarms (100\% specificity), while if the value is too low the system successfully detects all actual falls (100\% sensitivity) but, at the same time, may generate some false alarms (specificity $<100 \%$ ). This happens because several ADLs, like little jumps or fast sitting, are characterized by kinematic peaks similar to those of real falls. Thus, the overall detection accuracy of the system is a compromise between sensitivity and specificity.

2) Combining kinematic thresholds with posture: Some systems combine a fixed kinematic threshold with posture information to improve the detection accuracy. Li and Stankovic proposed a technique based on the idea that a fall always ends in a lying position [6]. Thus, a fall is detected when all the following three conditions are verified: $i$ ) the user is currently "static": acceleration amplitude in the last second is less than $0.4 \mathrm{~g}$; $i i)$ the current static posture is lying; iii) the alarm thresholds for both angular velocity and linear acceleration have been reached in the last 5 seconds.

The assumption that a fall always ends in a lying position allows to filter some fall-like ADLs like little jumps, quick sitting, and running. However, this assumption could lead to a number of false negatives because a patient may remain in a sitting position (for example against a wall [8]) after ground impact. Moreover, there may be some false positives when a person lies quickly on a bed or a sofa. According to their experimental evaluation, the sensitivity of this method is $91 \%$, while specificity is $92 \%$.

In general, approaches that make use of patients' posture need additional mechanisms to collect such information. If the position and orientation of the wearable device is fixed with respect to the patients' body, then posture can be determined by evaluating the orientation of the device with respect to the gravity. More information can be obtained by using two devices placed at different positions of the patients' body. For example, Table I shows which postures can be identified by using one or two sensors placed at user's chest and/or waist. If the sensor is attached to the user's chest, it is possible to understand if the user is standing/sitting or bending/lying, but it is not possible to discriminate between standing and sitting, and so on. The system described in [6] makes use of two devices equipped with a triaxial accelerometer and a gyroscope, which are placed at user's chest and right thigh.

\section{Motivation}

The solutions based on smart wearable devices equipped with motion sensors fully satisfy the requirements of automation and reactivity. Such systems also have the advantage of a fast and easy set-up, as well as the low costs required. However, the weak points of these approaches are represented by their usability and detection accuracy. Solutions based on a single device can be considered comfortable enough from the point of view of the monitored patient. Unfortunately, since it is not easy to gather relevant posture information using only one accelerometer, these systems generally relay only on a fixed threshold for the acceleration magnitude to detect falls, thus incurring into frequent and annoying false alarms (low specificity). On the other hand, attempts to improve accuracy, like the one based on postures proposed by Li et al. [6], generally require two or more devices and this is quite uncomfortable for patients. Moreover, when posture is inferred from data coming from two or more sensors, communication is required for their coordination which considerably reduces the lifetime of batteries.

We advocate the design of a fall detection system based on a single device able to filter false alarms without using posture information. The system we propose $i$ ) does not use gyroscopes, because they have a dramatic impact on battery lifetime [7]; $i i)$ uses a relatively low sampling frequency (sampling at $50 \mathrm{~Hz}$ seems to be a good trade-off between saving energy and collecting enough acceleration data about the fall-like event [5]); $i i i$ ) uses a single device placed at user's waist (waist is proved to offer the more relevant acceleration data [4] together with head and chest; however, waist seems to be the most comfortable position from patient's point of view); $i v$ ) does not use posture information (the validity of posture information strongly depends on the knowledge of the original orientation of the device; this information can be lost if, for example, a patient moves the device or after a violent fall; at the same time, if orientation is not considered, it is easier to wear the device); $v$ ) includes an innovative 
approach for the recognition and filtering of false alarms by using novel algorithms that use acceleration data to identify the most common fall-like ADLs.

\section{THE BASIC SYSTEM}

The system we implemented can be considered as a basic system, based on a single waist-mounted sensor, extended with a set of techniques able to filter the false alarms and increase its accuracy.

Even if the analysis of acceleration along the three different axes would provide more detailed information, the basic system uses only the magnitude of the acceleration vector. This choice is because the device is not completely integral with respect to the patient's body, and its orientation may change both at the time when it is put on or as a consequence of movements.

The basic system detects a fall-like event when the following conditions are satisfied: $i$ ) the magnitude of acceleration is greater than $3 g ; i i)$ the peak of acceleration magnitude is followed by a "static interval", which is a period of at least $1200 \mathrm{~ms}$ in which there are no peaks exceeding the threshold.

The $3 g$ value has been chosen so that, according to results obtained in [3] and in our collected data, the risk of false negatives is avoided, thus achieving a $100 \%$ degree of sensitivity. As already mentioned, there are several fall-like ADLs that reach this threshold, thus the specificity achieved is inevitably low. The static interval is used to understand when the previous event is finished, both when it is a fall-like ADL or when it is a real fall. After the detection of a fall-like event, the system tries to understand if the event is the consequence of an ADL or has been caused by a real fall. In the second case the system alerts the caregiver.

\section{RECOGNITION OF FALSE ALARMS}

The main contribution of our approach is the definition of a set of techniques able to filter false alarms without using posture information and thus making possible the adoption of fall detection systems based on a single accelerometer.

False alarms are recognized on the basis of peculiar patterns of the acceleration data. In a preliminary phase, we performed experiments to collect information about different types of real falls and common ADLs. In particular, we gathered the acceleration data of about 32 falls and 68 executions of different ADLs (the details of the data collection process, including the list of the different types of falls and ADLs, are presented in Section IV).

\section{A. Activities of daily living that may cause false alarms}

The analysis of the characteristics of ADLs and falls is fundamental for the development of filtering methods able to isolate false alarms from actual falls. The following ones are some classes of ADLs that can be confused with real falls and could generate false alarms:

A) sitting/lying quickly on soft/elastic surfaces (such as a bed or a sofa);

B) sitting quickly on medium/hard surfaces (such as a chair);
C) jumping on the ground.

The reason is that they present at least an acceleration magnitude peak which in some cases can be greater than $3 \mathrm{~g}$, which is the threshold used to detect a fall. Fortunately, each previously listed class of fall-like ADLs presents at least a feature that can be used to distinguish it from a real fall:

A) the fall-like ADLs happen on soft/elastic surfaces, thus they are characterized by smooth acceleration peaks;

B) it is distinguished by low/medium kinetic energy, which is quickly absorbed with a single sharp peak;

C) it has a typical acceleration shape, due to push, free fall and landing phases.

\section{B. Recognition of ADLs belonging to classes $A$ and $B$}

Falls are characterized by a violent impact on hard surfaces causing sharp peaks in the graph of acceleration magnitude. In general, the graph of acceleration magnitude contains several peaks (even if not all of them are greater than $3 \mathrm{~g}$ ) because of the following reasons: different parts of the body touch the ground at different times; the relatively high kinetic energy causes a sort of "rebound" effect on the body or parts of it. A sharp peak is characterized by quick variations of acceleration magnitude from a sample to the next. Differently, class A ADLs (sitting/lying on soft/elastic surfaces) present smooth peaks, since the kinetic energy is gracefully dissipated after impact, while class B ADLs (sitting on hard surfaces) generally determine a single sharp peak followed by quick stabilization. Therefore, there are relatively quick and numerous acceleration variations in falls, slow variations in class $\mathrm{A}$ ADLs and few variations in class B ADLs.

In order to extract the features previously described from raw acceleration data, we defined a measure, the Average Acceleration Magnitude Variation index, defined as follows:

$$
A A M V=\sum_{i \in W} \frac{\left|a c c_{i+1}\right|-\left|a c c_{i}\right|}{\text { number of samples in } W}
$$

The value is computed in a time window $(W)$ of proper size which includes the $3 g$ peak. Through an empirical evaluation we found that the AAMV window which provides the best results, according to our dataset, is the one that starts $640 \mathrm{~ms}$ before the last $3 \mathrm{~g}$ peak and ends $540 \mathrm{~ms}$ after the peak. The value of AAMV is directly proportional not only to how quickly the acceleration magnitude changes, but also to the number of peaks present in the considered window. As a consequence, we expect to find greater AAMV values for real falls with respect to those obtained for ADLs belonging to classes A and B. Figures 1a, 1b, 1c, and 1d show the typical acceleration graphs of examples of real falls and ADLs belonging to classes $\mathrm{A}$ and $\mathrm{B}$. The AAMV window is also shown. We performed a binary classification of data and, as expected, the AAMV values obtained for ADLs belonging to class A and B are lower than those obtained for real falls. This difference is clearly shown in Fig. 2. By comparing the AAMV of the potential fall with a threshold (approximately $0.27 \mathrm{~g}$ ) it is possible to classify the event as a real fall, or as an ADL belonging to class A or B. In the latter case, even if the acceleration magnitude exceeds the $3 g$ threshold, the fall-like 
Acceleration magnitude VS time

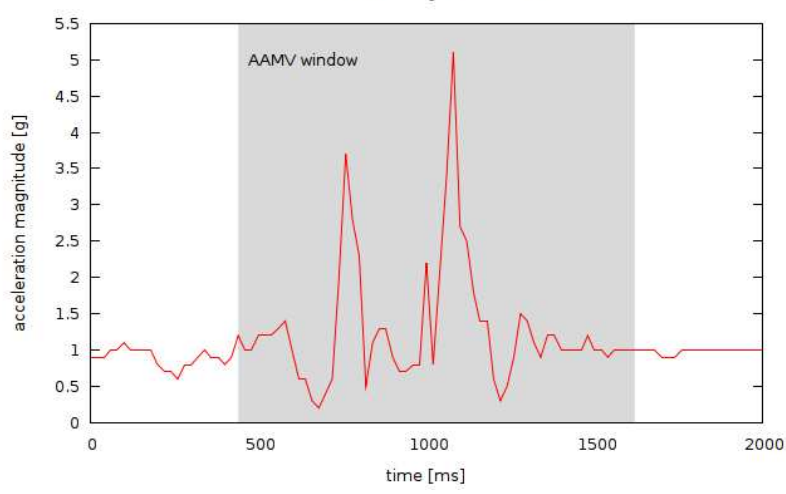

(a) Real fall

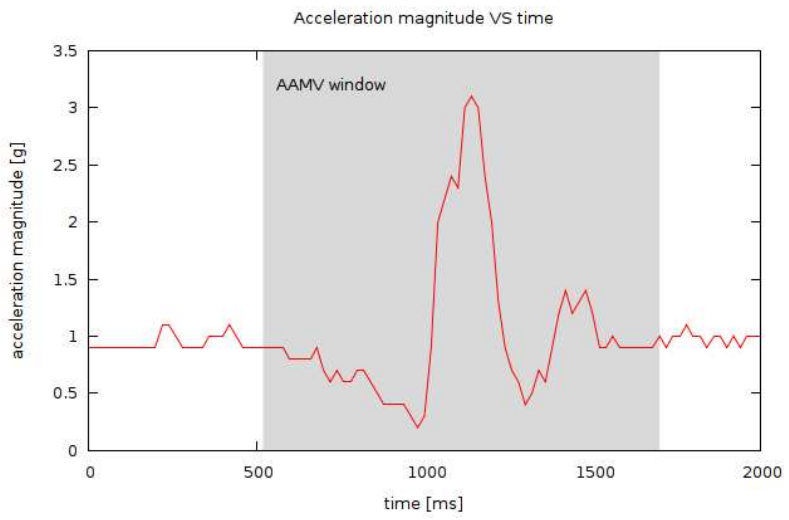

(c) Class A (sitting/lying quickly on a soft surface)

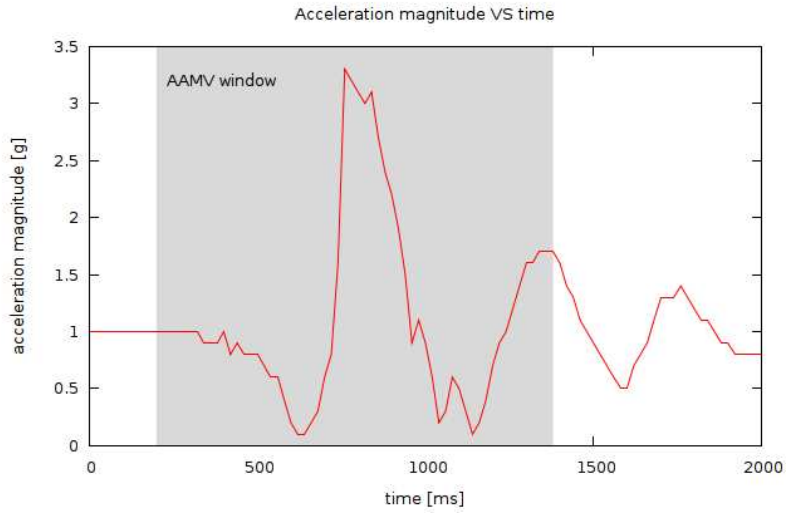

(b) Class A (sitting/lying quickly on a elastic surface)

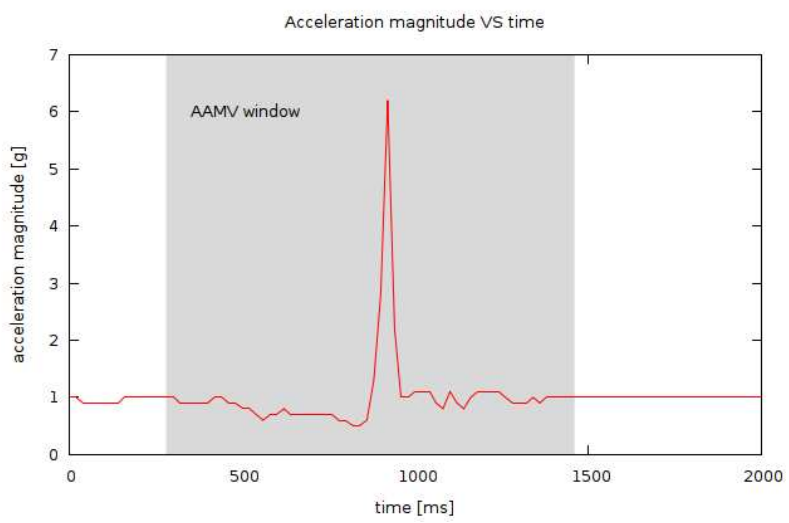

(d) Class B (sitting quickly on a medium/hard surface)

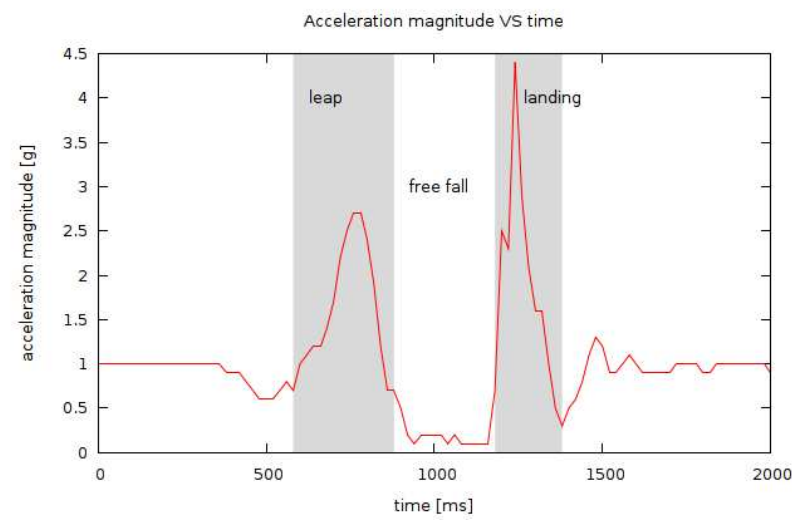

(e) Class C (jumping)

Fig. 1. Acceleration for real falls and ADLs belonging to classes A, B, and C

event is filtered and an alarm is not raised, thus increasing the specificity of the system.

\section{Recognition of ADLs belonging to class $C$}

The filtering method based on the AAMV index cannot be applied successfully to class C fall-like ADLs. The reason of this can be explained considering Figure 1e, which shows a typical acceleration magnitude graph obtained performing a small jump on the floor. There are two relevant peaks: the first is produced when the user leaps, the second (about $4.5 \mathrm{~g}$ ) is higher and sharper and corresponds to the landing on the floor. These variations determine AAMV values for jumps that are comparable to those obtained for real falls.

Jumping consists of three phases: leap, free fall, landing. As shown in Figure 1e, each phase can be easily identified in the acceleration magnitude graph. This peculiar shape represents the feature that can be used to filter this class of false alarms. Recognition of a jump can be performed through the following procedure:

1) Verify the presence of a peak associated with the leap.

2) Find two instants 


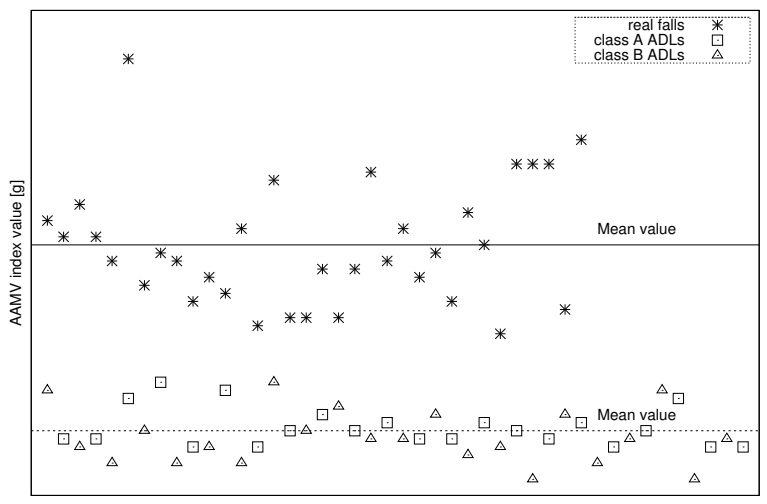

Fig. 2. Values of the AAMV index for real falls and ADLs belonging to classes $\mathrm{A}$ and $\mathrm{B}$

\begin{tabular}{|c|c|c|c|c|}
\hline User ID & Sex & Age & Height cm & Weight kg \\
\hline U1 & male & 24 & 175 & 62 \\
\hline U2 & male & 37 & 177 & 81 \\
\hline U3 & male & 26 & 178 & 75 \\
\hline U4 & male & 64 & 175 & 91 \\
\hline
\end{tabular}

TABLE II

USERS INVOLVED IN THE DATA COLLECTION.

a) landing start: $80 \mathrm{~ms}$ before the last acceleration magnitude peak greater than $3 \mathrm{~g}$. This is a empirical estimation of when landing begins.

b) leap end: found searching backwards in time from $20 \mathrm{~ms}$ before landing start until measured acceleration magnitude is greater than or equal to $1 \mathrm{~g}$. This is a simple estimation of when free fall begins.

3) Use these instants to find two quantities:

a) Free Fall Interval (FFI): as the difference between landing start and leap end.

b) Free Fall Average Acceleration Magnitude (FFAAM): average acceleration magnitude in the free fall interval.

We noticed that real falls have a lower FFI or a higher FFAAM value with respect to the values obtained for jumps. Thus, we defined two conditions useful to recognize an event as a class C ADLs:

- $F F I>100 \mathrm{~ms}$

- $F F A A M<0.5 g$

An event is classified as a class C ADL only if both tests are passed. In such case, an alarm is not raised.

\section{COLleCtion OF DATA AND IMPLEMENTATION}

In this section we describe the acquisition of data related to real falls and different ADLs. We also provide some details about the implementation of the system.

\section{A. Data collection}

Data acquisition is the first step in every fall detection study and it is a time-consuming process. Accelerations measured during tests are fundamental to understand the features that can be used to isolate falls from harmless actions like sitting

\begin{tabular}{|l|c|}
\hline Action & Short form \\
\hline Jumping & JUM \\
\hline Lying quickly on bed & LBE \\
\hline Lying quickly on a mat & LMA \\
\hline Lying quickly on sofa & LSO \\
\hline Parkinsonian gait & PGA \\
\hline Running & RUN \\
\hline Sitting quickly on armchair & SAR \\
\hline Sitting quickly on chair & SCH \\
\hline Sitting quickly on sofa & SSO \\
\hline
\end{tabular}

TABLE III

LIST OF FALL-LIKE ADLS.

\begin{tabular}{|l|c|}
\hline Action & Short form \\
\hline Fall from bed & FBE \\
\hline Fall almost vertically from standing (faint) & FFA \\
\hline Fall after parkinsonian gait & FPG \\
\hline Fall forward landing on hands first & FHA \\
\hline Fall after a small jump & FJU \\
\hline Fall forward landing on knees first & FKN \\
\hline Fall while running & FRU \\
\hline Fall from sitting & FSI \\
\hline
\end{tabular}

TABLE IV

TYPES OF FALLS PERFORMED.

or lying. Unfortunately, previous studies generally describe the performed tests and the obtained results, but the acceleration data is usually not made available.

Our experiments involved four male subjects. They have been engaged into a battery of tests designed to collect data about the most common fall-like ADLs and falls. Recorded ADLs always present at least an acceleration magnitude peak greater than $3 \mathrm{~g}$, which is followed by a static interval lasting at least $1200 \mathrm{~ms}$. These are the kinds of ADLs that would produce a false alarm in the basic fall detection system. Skate pads have been used to avoid injuries to knees, elbows and wrists, since landing always took place on hard surfaces. This also ensured a realistic execution of falls as it removed the fear of hitting the ground (and thus reduced the effects of those semi-unconscious actions aimed at self-protection in planned falls). Table II shows the profiles of the volunteers who have been involved in the collection of data. Table III describes the list of fall-like ADLs, while Table IV shows the list of real falls. In fact, different types of falls could be defined, each characterized by a peculiar way of landing on the floor, or by the action performed before losing balance. Finally, Tables $\mathrm{V}$ and VI respectively show the number of ADLs and the number of falls performed by each volunteer.

Note that we decided to collect, represent, and store also the information not used in our method for the recognition of false alarms, such as the posture of the user before and after a potential fall or the separate acceleration values along the three axes (we used only the magnitude). This has been done to foster the reuse of the collected data in future work and to enable the evaluation of future techniques on the same set of data $^{1}$.

\footnotetext{
${ }^{1}$ The database will be made available via Web and open without any access restriction in case of publication of this paper.
} 


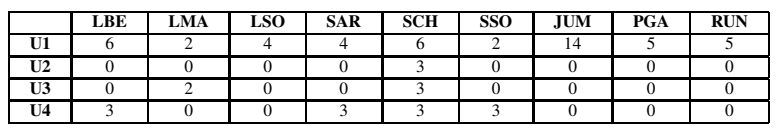

TABLE V

FALL-LIKE ADLS PERFORMED BY EACH USER.

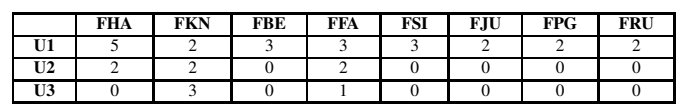

TABLE VI

FALLS PERFORMED BY EACH VOLUNTEER

\section{B. Implementation}

We used a Shimmer mote as the wearable device [1]. Shimmer is a wireless sensor platform, characterized by a small form factor, that can record and transmit physiological and kinetic data in real-time. The chosen device incorporates a triaxial accelerometer, a microcontroller and a radio transceiver. The output of the accelerometer is sampled by the microcontroller at a $50 \mathrm{~Hz}$ frequency. Another mote is used to transform a generic PC into a base station. It is connected via the USB port to the $\mathrm{PC}$ and communicates using the radio with the wearable device, acting as a packet forwarder. The base station displays an alarm message to the caregiver when an alarm packet is received from the wearable device. There are two ways of implementing the filtering technique. The first consists of using the embedded intelligence of the wearable device. The main advantage of this approach is that several useless transmissions to the base station can be avoided by filtering the false alarms directly on the remote device, enhancing the lifetime of batteries. The second consists in running the algorithm on the base station, after the acceleration samples of the event have been received via radio. The advantage of this approach is the abundance of computing resources on the base station. Since the extraction of AAMV, FFI, and FFAAM values from acceleration data is not computationally intensive, we implemented the filtering techniques directly on the wearable device in order to communicate with the base station only when an alarm occurs. Software has been developed using the TinyOS/nesC platform.

\section{RESULTS AND CONCLUSION}

The collected data has been used to evaluate the performance of our techniques for the recognition of false alarms. As previously mentioned in Section III-B, in the data we collected, the set of AAMV values of real falls does not overlap with the set of AAMV values of ADLs belonging to classes A and B. This allowed us to filter all the false alarms coming from such activities without missing any real fall. Similarly, all the ADLs belonging to class $\mathrm{C}$ of our collected data satisfy the conditions described in Section III-C, while the real falls fail both of them. Henceforth, these filtering methods achieve $100 \%$ sensitivity and specificity values with respect to the data we collected.

Despite the relatively small data set, the prototype proves that the idea of filtering ADLs on the basis of peculiar features of the acceleration data can be used to enhance significantly the specificity of a basic fall detection system. We applied this idea to a system based on just one accelerometer placed at patient's waist. However, the same idea could be adapted to improve the accuracy of systems based on two or more devices, or placed at a different position of the patient's body, after proper analysis of the acceleration data and the extraction of new peculiar features. It is also important to notice that the enhancements in terms of detection accuracy have been achieved without compromising the usability of the system.

Certainly we do not believe to have definitely solved the fall detection problem, since more falls and fall-like events have to be studied in order to refine the filtering algorithms. However, from our results we have reasons to believe that the proposed approach can actually facilitate the development of fall detection systems with an improved level of accuracy and usability.

\section{REFERENCES}

[1] http://www.shimmer-research.com.

[2] A. K. Bourke and G. M. Lyons. A threshold-based fall-detection algorithm using a bi-axial gyroscope sensor. Medical engineering \& physics, 30(1):84-90, 2008

[3] A. K. Bourke, J. V. O'Brien, and G. M. Lyons. Evaluation of a thresholdbased tri-axial accelerometer fall detection algorithm. Gait \& posture, 26(2):194-9, 2007.

[4] Maarit Kangas, Antti Konttila, Ilkka Winblad, and Timo Jämsä. Determination of simple thresholds for accelerometry-based parameters for fall detection. In Proceedings of the Annual International Conference of the IEEE Engineering in Medicine and Biology Society, pages 136770, January 2007

[5] Dean M Karantonis, Michael R Narayanan, Merryn Mathie, Nigel H Lovell, and Branko G Celler. Implementation of a real-time human movement classifier using a triaxial accelerometer for ambulatory monitoring. IEEE Transactions on Information Technology in Biomedicine, 10(1):156-67, January 2006.

[6] Qiang Li, John A. Stankovic, Mark A. Hanson, Adam T. Barth, John Lach, and Gang Zhou. Accurate, fast fall detection using gyroscopes and accelerometer-derived posture information. In International Workshop on Wearable and Implantable Body Sensor Networks, pages 138-143, Berkeley, CA, USA, 2009. IEEE Computer Society.

[7] Konrad Lorincz, Bor-rong Chen, Geoffrey Werner Challen, Atanu Roy Chowdhury, Shyamal Patel, Paolo Bonato, and Matt Welsh. Mercury: A Wearable Sensor Network Platform for High-Fidelity Motion Analysis. In Proceedings of the 7th ACM Conference on Embedded Networked Sensor Systems (SenSys'09), Berkeley, CA, 2009.

[8] N. Noury, P. Barralon, G. Virone, P. Boissy, M. Hamel, and P. Rumeau. A smart sensor based on rules and its evaluation in daily routines. In Proceedings of the 25th Annual IEEE International Conference on Medicine and Biology Society, pages 3286-3289, 2003.

[9] N Noury, A Fleury, P Rumeau, A K Bourke, G O Laighin, V Rialle, and $\mathrm{J}$ E Lundy. Fall detection-principles and methods. In Conference Proceedings of the Annual International Conference of the IEEE Engineering in Medicine and Biology Society, pages 1663-6, 2007.

[10] Xinguo Yu. Approaches and principles of fall detection for elderly and patient. In 10th IEEE International Conference on e-Health Networking, Applications and Services (HealthCom 2008), pages 42-47, 2008. 